\title{
Afterglow of a Microwave Microstrip Plasma as an Ion Source for Mass Spectrometry
}

\author{
Kevin P. Pfeuffer, Allen White ${ }^{\dagger}$, Jose A.C. Broekaert ${ }^{\ddagger}$, Gary M. Hieftje* \\ Department of Chemistry, Indiana University, Bloomington, Indiana, USA, \\ $\dagger$ Permanent Address: Rose-Hulman Institute of Technology, Terre Haute, Indiana, USA \\ \$ On Leave From: Institute for Inorganic and Applied Chemistry, University of Hamburg, \\ Hamburg, Germany
}

*Corresponding Author: Tel: (812) 855-2189, Fax: (812) 855-0958, e-mail:Hieftje@indiana.edu 


\begin{abstract}
:
A microwave-induced plasma that was previously used for optical emission spectrometry has been repurposed as an afterglow ion source for mass spectrometry. This compact microwave discharge, termed the microstrip plasma (MSP), is operated at 20-50 W and $2.45 \mathrm{GHz}$ in helium at a flow of $300 \mathrm{~mL} / \mathrm{min}$. The primary background ions present in the afterglow are ionized and protonated water clusters. An exponential dilution chamber was used to introduce volatile organic compounds into the MSP afterglow and yielded limits of detection in the $40 \mathrm{ppb}$ to 7 ppm range $(\mathrm{v} / \mathrm{v})$. A hydride-generation system was also utilized for detection of volatile hydride-forming elements (arsenic, antimony, tin) in the afterglow and produced limits of detection in the 10-100 ppb range in solution. The MSP afterglow was found capable of desorption and ionization of analyte species directly from a solid substrate, suggesting its use as an ion source for ambient desorption/ionization mass spectrometry.
\end{abstract}

Keywords: Microwave plasma, Hydride-Generation, Ambient Desorption/Ionization, Miniaturization, Volatile Organic Compounds

\title{
1. Introduction:
}

Rare-gas plasmas have a strong history as excitation/ionization sources for optical emission (OES) and mass spectrometric (MS) analysis. The most well known plasma source for elemental analysis is the radio-frequency inductively coupled plasma (ICP), developed in the $1960 \mathrm{~s}^{1}$ and patented in $1969^{2}$. Higher frequencies were also found useful for atomic analysis in the form of a microwave plasma, which operated at frequencies above $300 \mathrm{MHz}$, typically 2.45 $\mathrm{GHz}^{3}$. The microwave plasma has some advantages over an ICP, including its ability to be operated at moderate power levels and gas flows, even in helium.

Ambient desorption/ionization mass spectrometry (ADI-MS), begun with the introduction of desorption electrospray ionization (DESI) in $2004^{4}$, showcases the ability of traditional ionization sources to be used in alternative ways. In this case it was found that addition of an auxiliary gas flow around an electrospray ionization (ESI) ${ }^{5}$ source could generate ions directly from a sample surface. As an extension of this thinking, an atmospheric-pressure glow discharge (APGD) was operated in the flowing afterglow mode and generated ions directly from a sample surface. ${ }^{6,7}$ Similarly, a heater was added to a corona discharge to create the direct

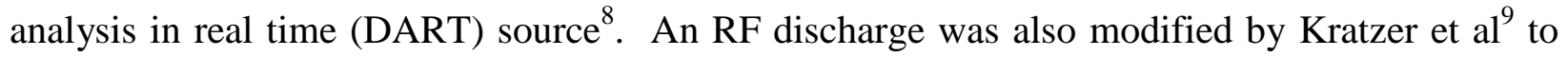
function as an ADI-MS source. From these examples, it seems inevitable that a microwave discharge would be applied to ambient mass spectrometry ${ }^{10,11}$.

Miniaturization has increased the attractiveness of sources for use under ambient conditions. Examples include a liquid-sampling glow discharge ${ }^{12}$, and a microfabricated atmospheric-pressure glow discharge ${ }^{13}$. A compact microwave plasma source would be particularly well suited for ADI-MS. Such a source, the microwave microstrip plasma (MSP), 
was introduced by the Broekaert group in $1999^{14,15}$ and improved in $2003^{16}$. The source was used primarily for optical emission spectrometry (OES) of inorganic species, including the coldvapor technique for the determination of mercury ${ }^{17-19}$, hydride generation for the determination of arsenic and antimony ${ }^{20,21}$, as a detector for halogenated hydrocarbons in gas chromatography, ${ }^{22}$ and for gas-phase bromine, chlorine, sulfur, carbon dioxide and cyanide determination $^{23}$. However, these applications all involved introduction of sample material into the microwave discharge itself, which can perturb the plasma. In the present study, the afterglow of the MSP discharge is utilized for sample ionization and desorption, in order to avoid plasma quenching and memory effects. Ambient desorption/ionization, exponential dilution and hydride generation were all explored for sample introduction into the MSP afterglow and yielded attractive figures of merit.

\section{Experimental:}

Figure 1 displays a schematic diagram of the microstrip plasma (MSP) and a white-light image of the MSP at the inlet of an atmospheric-sampling mass spectrometer. All samples were introduced into the region between the MSP and the entrance orifice of the mass spectrometer. The distance between the microwave source and the inlet was varied to determine optimal placement, based on signal level, and was found to be $5 \mathrm{~mm}$.

The MSP has been extensively described previously ${ }^{14-16}$, but will be recapped here for clarity. A square piece of sapphire $(30 \times 30 \times 1.2 \mathrm{~mm})$ is used to support the MSP, with a $0.9 \mathrm{~mm}$ diameter hole through the center for gas delivery. Copper strips deposited on top $(0.8 \mathrm{~mm}$ wide strip) of the plate distribute power for the discharge; a balancing arm was added for impedance matching. Power was supplied through a microwave power generator (Microtron 200, Electro Medial Supplies Ltd, Wantage, Oxfordshire, England) through a double-stub tuner ( Model DSI09M, Weinschel Engineering, Gaithersburg, MD, USA), which was adjusted to keep reflected power below 5 watts. The input line was terminated in a stub resistor (Model 8135, Bird Electronic Corporation, Cleveland, USA). All gases were ultra-high purity (99.999\%, Airgas mid-America, Bowling Green, Kentucky, USA). Helium flow was adjusted by a mass flow controller (MKS instruments, Andover, MA, USA). All analytes were reagent-grade

A commercial time-of-flight mass spectrometer was used (LECO Renaissance ${ }^{\circledR}$, LECO Corporation, St. Joseph, MI). Modifications to the instrument have been described in depth previously ${ }^{24}$, but will be briefly covered here. A roughing pump was added to the first stage of the spectrometer along with an atmospheric-pressure interface which consisted of a steel plate with a $250-\mu \mathrm{m}$ diameter aperture. An ion lens was added in the first vacuum stage to improve ion throughput into the second stage of the mass spectrometer interface.

A continuous-flow hydride-generation system was constructed according to previous experimental setups ${ }^{25,26}$. Sodium borohydride solutions stabilized with sodium hydroxide (both $1 \%$ in weight) were mixed with the analyte solution in a hydrochloric or nitric acid matrix (each 
$1 \mathrm{M}$ ); flow rates were controlled with a peristaltic pump (Gilson, Middleton, WI) into a mixing tee at a flow rate of $0.5 \mathrm{~mL} / \mathrm{min}$. To ensure complete mixing, a reaction coil was made from a $100-\mathrm{cm}$ length of PTFE tubing. Liquid waste and analyte hydride passed into a gas-liquid separator that was filled with 2-mm diameter glass beads to increase the surface area and allow complete reaction to occur. A supplementary gas flow of helium at $50 \mathrm{~mL} / \mathrm{min}$ was set by a mass flow controller (MKS instruments, Andover, MA, USA). The helium flow, which contained volatile hydride species, was introduced into the afterglow through a glass capillary (1 $\mathrm{mm}$ I.D.) positioned between the MSP and the inlet of the mass spectrometer. The position of the capillary was controlled with an $\mathrm{x}, \mathrm{y}, \mathrm{z}$ fiber-optic stage (Newport Corporation, Irvine, CA).

An exponential dilution chamber (EDC $)^{27}$ was also utilized to determine limits of detection for volatile organic compounds. The experimental setup has also been described previously $^{24}$. In brief, a well-stirred $100 \mathrm{~mL}$ three-neck round-bottomed flask was used as the dilution chamber and helium flow through the flask was regulated by a mass flow controller (MKS instruments, Andover, MA, USA). The same glass capillary/stage setup used for hydride generation was employed to deliver the helium and analyte vapor to the optimized (based on signal level) position between the MSP and the mass spectrometer.

Headspace from volatile organic compounds (200-1000 $\mu \mathrm{g})$ was injected by means of a GC syringe into the round bottom flask. Based on the vapor pressure of the analyte, the amount injected, and the volume of the flask, an initial concentration was calculated. Then, from an exponential decay equation, the time domain signal was transformed into the concentration domain, to enable a calibration curve to be generated.

Powdered solid samples were introduced into the afterglow stream on melting-point capillaries (Chemglass Life Sciences, Vineland, NJ, USA) mounted on a custom-built stage (Newport corporation, Irvine, CA, USA). Simple mechanical abrasion was used to transfer small amounts $(<1 \mathrm{mg})$ of material from a solid sample onto the melting-point capillary and into the MSP afterglow.

\section{Results and Discussion:}

Helium and argon were both used previously with the MSP. However, the current geometry of the MSP could be ignited only in helium. Examination of the chip showed significant erosion of the sapphire and copper at the exit of the strip. This erosion is believed to impact the chip's ability to sustain a discharge. Accordingly, helium was used for all studies reported here. The signal for the protonated water cluster dimer $\left(\mathrm{H}_{2} \mathrm{O}\right)_{2} \mathrm{H}^{+}(\mathrm{m} / z$ 37) was used for optimization. Helium flow was found to be optimal at $300 \mathrm{~mL} / \mathrm{min}$ and applied power at $50 \mathrm{~W}$; these conditions were used for all subsequent experiments. 
A background mass-spectral scan is shown in Figure 2. As with the microwave sources for ambient mass spectrometry described by Zhang et. al. ${ }^{10}$ protonated water clusters are prominent at $m / z 19,37,55,72$, etc.. However, we disagree with the earlier ${ }^{10}$ assignment of the even-mass peaks seen one $\mathrm{m} / \mathrm{z}$ lower than the water clusters $(\mathrm{m} / \mathrm{z} 18,36,54,71 \mathrm{etc}$.). These peaks were earlier asserted to be ammoniated water clusters (e.g. $\mathrm{H}_{2} \mathrm{O} \cdot \mathrm{NH}_{4}{ }^{+}$for $\mathrm{m} / \mathrm{z} / 36$ ). In contrast, we believe these species are simply ionized water clusters as labelled in Figure 2. Zhang et. al. ${ }^{10}$ based their assignment of ammonia clusters on spectra of acetone and acetic acid, which were present only as the ammoniated $\left(\mathrm{M}+\mathrm{NH}_{4}\right)^{+}$peak; however, we saw only protonated molecular ions for acetone (Table 1) and acetic acid (data not shown). Literature cited by Zhang et. al. ${ }^{10}$ indicates that ammonia can be produced within microwave plasmas but exclusively for $\mathrm{N}_{2} / \mathrm{H}_{2}$ plasmas, ${ }^{28}$ which differ strongly from helium or argon plasmas or with nitrogen interacting with the afterglow of such plasmas. Additionally, other studies utilizing He plasmas have indicated that $\mathrm{NH}_{4}{ }^{+}$is not produced ${ }^{29,30}$ and is unstable at atmospheric pressure ${ }^{31}$. Based on this evidence we conclude that ionized water clusters are a more likely assignment for this even-mass series. Also, unlike some other plasma-based sources ${ }^{24}$ the MSP produces very little $\mathrm{NO}^{+}$. As a result, ionization arises primarily from proton transfer although evidence of charge-transfer can be found in the form of some molecular ions (cf. Table 1).

The distribution between ionized and protonated water clusters shifts as the cluster size increases (Figure 2). For monomer and dimer water clusters, ionized molecular species $(\mathrm{m} / \mathrm{z} / 18$ and 36) are dominant, but for the third through fifth clusters the protonated water clusters $(\mathrm{m} / \mathrm{z} /$ 55 and 72) are the most abundant. It is not currently known why this shift between direct ionization and protonation occurs.

\subsection{Organic-Vapor Introduction from an Exponential Dilution Chamber}

In early experiments, introduction of organic vapors directly into the MSP discharge channel in the sapphire plate proved unsuccessful; the discharge was either extinguished, or no analyte signal was seen. Consequently, a glass capillary was used to carry effluent from the exponential dilution chamber into the afterglow, in the $5 \mathrm{~mm}$ gap between the MSP and the inlet to the mass spectrometer. Exponential dilution relies on a well-mixed container that produces a first-order (exponential) decay in concentration upon injection of a spike of analyte. An example of this exponential decay for protonated acetonitrile can be seen in Figure 3a. The injection of acetonitrile vapor into the stirred flask occurred at 60 seconds and the afterglow signal showed excellent time response with a rise time of $17 \mathrm{~s}$ (based on 90/10 criteria) and a delay of 30s from injection to $90 \%$ maximum signal. A calibration curve generated from the exponential decay in Figure $3 \mathrm{a}$ is shown in Figure $3 \mathrm{~b}$. Significant roll-off occurs at high concentrations ( $<40 \mathrm{ppb} v / \mathrm{v}$ ), probably because of depletion of reagent ions in the afterglow ${ }^{24}$. As a result of the roll-off, the low concentration region ( $c f$. inset in Figure $3 b$ ) of the calibration plot was used for quantitation. A 3- $\sigma$ approach was used for LODs, with the time trace before analyte injection used as the blank. 
Limits of detection, amount injected and the ion observed for a variety of volatile organic compounds are compiled in Table 1. LODs are in the range of 0.04-7 ppm (v/v), with the best values obtained for compounds that formed protonated ions. The low sensitivity for molecular ions (benzene and chloroform) is likely because they are formed through a charge-transfer mechanism that requires reagents such as $\mathrm{NO}^{+}$, which are present in low concentration in this source (cf. Figure 2). Ethyl acetate was detected as the protonated molecular ion, yet exhibited a relatively high LOD, likely due to significant fragmentation that occurred either during ionization or in the first stage of the mass spectrometer interface ${ }^{32}$.

\subsection{Hydride Generation}

Volatile hydrides were introduced into the afterglow of the MSP through the same glass capillary used in the exponential dilution work. Spectra for $100 \mathrm{ppm}$ arsenic and a blank are shown in Figure 4. The main peaks for the arsenic-containing solution are oxygen, water and hydroxide adducts with As, although a small peak is seen for $\mathrm{AsH}_{2}{ }^{+}$. Similar clustering has been observed previously for introduction of hydrides into the open atmosphere ${ }^{25}$. The prominent peak of $\mathrm{AsO}\left(\mathrm{H}_{2} \mathrm{O}\right)^{+}$was used for quantification purposes. Clustering of arsenic with water and oxygen $^{24}$ was found to be problematic for high concentrations of As, and causes roll-off in the calibration curve in Figure 5. The consistency of roll-off was evaluated by running the highest concentration $(10 \mathrm{ppm})$ in triplicate; all runs overlapped. The temporal characteristics of this setup are displayed in Figure 6. Rise and fall times were determined based on 90/10\% consideration; the rise time was $30 \mathrm{~s}$ while the fall time was $50 \mathrm{~s}$. The longer fall time is likely due to the large void volume created by the glass beads in the gas-liquid separator, which leads to significant tailing of the analyte signal. Signal drift is apparent between the rise and fall portions of the washout curve. From 200-450 seconds the trace in Figure 6 remains stable, while a noticeable decrease is seen from 450-700 seconds. The reason for this decline in signal is not currently known, but is possibly due to a shift in mechanical alignment between the capillary and afterglow. This drift is significant at $2 \%$ /minute (based on maximum value) and care should be taken to ensure it does not compromise future experiments. All calibrations reported here were for one minute (with one-second integration time) and no significant drift was observed.

Detection limits are listed in Table 2 for tin, arsenic and antimony and range from 36-120 ppb in solution. These limits of detection are deemed suitable for screening applications, especially in an ambient fashion. However, previous detection limits for hydride generation with an ADI-MS source were in the single ppb range ${ }^{25}$ and those obtained in conventional glowdischarge TOFMS ${ }^{33}$ are 100 times better. This difference is likely due to a combination of the molecular clustering seen in mass spectra, which distributes the analyte among several massspectral peaks, and the use of ambient sample introduction, which is inherently somewhat inefficient. 


\subsection{Noise Power Spectra}

Noise power spectra (NPS) were obtained for both the exponential-dilution chamber and the hydride-generation systems ( $c f$. Figure $7 \mathrm{a}$ and $\mathrm{b}$, respectively). For the exponential-dilution power spectrum the protonated water dimer was monitored, whereas the arsenic oxide water adduct $\left(\mathrm{AsO}\left(\mathrm{H}_{2} \mathrm{O}\right)^{+}\right)$was tracked for hydride generation. Because the time-of-flight mass spectrometer had a minimum integration time of $12.5 \mathrm{~ms}(80 \mathrm{~Hz})$, the NPS were Nyquist-limited to $40 \mathrm{~Hz}$. The strongest periodic component occurred at $20 \mathrm{~Hz}$, for both sample-introduction approaches, which suggests it originates from a source other than the sample-introduction system. The most likely source is aliased $60 \mathrm{~Hz}$ noise, which has been observed previously for a hydride generation system ${ }^{25}$. Noise spectra taken with and without the exponential dilution chamber and the hydride generation system in place show that no significant noise features come from either setup (data not shown). Overall, the hydride generation yielded lower random noise levels $(\mathrm{dB})$ than the exponential-dilution setup which is perhaps why drift ( $1 / f$ noise) is more apparent in Figure $7 \mathrm{~b}$ than in Figure 7a.

\subsection{Solid Sample Analysis}

A small amount $(<1 \mathrm{mg})$ of material was mechanically transferred from a TopCare ${ }^{\circledR}$ acetaminophen tablet to the tip of a melting point capillary. The capillary tip was then inserted into the afterglow between the MSP and the front plate of the mass spectrometer. Time traces for protonated acetaminophen $(\mathrm{m} / \mathrm{z}, 152)$ and the first two water-cluster reagent ions $\left(\left(\mathrm{H}_{2} \mathrm{O}\right) \mathrm{H}^{+},\left(\mathrm{H}_{2} \mathrm{O}\right)_{2} \mathrm{H}^{+}, m / z, 19\right.$ and 37, respectively) are shown in Figure 8. Upon introduction of the probe into the after glow (at 30s) there is an initial peak for the protonated molecular ion that quickly disappears (FWHM 5s) and does not return, which suggests the sample is either totally consumed or lost from the probe by the helium flow and not ionized. However, there is an attenuation of both water clusters upon insertion of the melting point capillary that lasts longer than the protonated acetaminophen signal. The initial depletion of reagent ions correlates temporally with the protonated analyte signal and is due to reagent-ion depletion. However, after the analyte signal returns to baseline, the reagent-ion signals remains attenuated until the probe is removed (after 140 seconds). This persistent signal attenuation suggests that some of the helium flow is deflected, so reagent ions from the MPC afterglow do not reach the MS inlet. This behavior has been observed previously for plasma-based ambient mass spectrometry systems ${ }^{34}$. Upon removal of the probe (after 140s) the water-cluster signal returns to its previous level.

\section{Conclusions:}

The afterglow of a microwave microstrip plasma (MSP) is an attractive ion source for several types of mass spectrometry. A background ion scan indicated that the primary reagent ions are ionized and protonated water clusters. Monitored analyte ions were typically either 
protonated or charged molecule ions, which suggests that the dominant ionization mechanisms are charge or proton transfer, in common with most discharge-based ADI-MS sources. Limits of detection ranged from $40 \mathrm{ppb}$ to $7 \mathrm{ppm}$ (vol/vol) for volatile organic compounds introduced via an exponential dilution system. Volatile hydride-forming elements could be introduced through a hydride-generation system, with LODs in the range of 10-100 ppb in solution. Finally, it was shown that the MSP afterglow is capable of desorption and ionization directly from a solid sample.

\section{Acknowledgements:}

Supported by the U.S. Department of Energy through grant FG02-98ER14980. The authors wish to thank the LECO corporation for the generous loan of the Renaissance ${ }^{\circledR}$ TOFMS instrument. José A. C. Broekaert would like to acknowledge the Alexander-von-HumboldtFoundation (Bonn, Germany) for travel support. Dr. White is supported in part by an RUI under NSF grant no. 1062846.

\section{References:}

[1] S. Greenfield, I. L. Jones and C. T. Berry, High-pressure plasmas as spectroscopic emission sources, Analyst 89 (1964), 713-720

[2] B. C. Thomas, G. Stanley and J. I. L. William, Pat. No.: US3467471 A, 1969.

[3] S. Greenfield, H. M. McGeachin and P. B. Smith, Plasma emission sources in analytical spectroscopy-II, Talanta 22 (1975), 553-562

[4] Z. Takats, J. M. Wiseman, B. Gologan and R. G. Cooks, Mass Spectrometry Sampling Under Ambient Conditions with Desorption Electrospray Ionization, Science 306 (2004), 471-473

[5] J. Fenn, M. Mann, C. Meng, S. Wong and C. Whitehouse, Electrospray ionization for mass spectrometry of large biomolecules, Science 246 (1989), 64-71

[6] F. J. Andrade, G. Gamez, M. R. Webb, W. C. Wetzel, S. J. Ray and G. M. Hieftje, presented in part at the Pittcon, Orlando, March 12-17, 2006, 2006.

[7] F. J. Andrade, W. C. Wetzel, G. C. Y. Chan, M. R. Webb, G. Gamez, S. J. Ray and G. M. Hieftje, A new, versatile, direct-current helium atmospheric-pressure glow discharge, J. Anal. At. Spectrom. 21 (2006), 1175-1184

[8] R. B. Cody, J. A. Laramée and H. D. Durst, Versatile New Ion Source for the Analysis of Materials in Open Air under Ambient Conditions, Anal. Chem. 77 (2005), 2297-2302

[9] J. Kratzer, Z. Mester and R. E. Sturgeon, Comparison of dielectric barrier discharge, atmospheric pressure radiofrequency-driven glow discharge and direct analysis in real time sources for ambient mass spectrometry of acetaminophen, Spectrochim. Acta Part B 66 (2011), 594-603 
[10] T. Zhang, W. Zhou, W. Jin, J. Zhou, E. Handberg, Z. Zhu, H. Chen and Q. Jin, Direct desorption/ionization of analytes by microwave plasma torch for ambient mass spectrometric analysis, J. Mass Spec. 48 (2013), 669-676

[11] X. Zhan, Z. Zhao, X. Yuan, Q. Wang, D. Li, H. Xie, X. Li, M. Zhou and Y. Duan, Microwave-Induced Plasma Desorption/Ionization Source for Ambient Mass Spectrometry, Anal. Chem. 85 (2013), 4512-4519

[12] R. K. Marcus, C. Burdette, B. Manard and L. Zhang, Ambient desorption/ionization mass spectrometry using a liquid sampling-atmospheric glow discharge (LS-APGD) ionization source, Anal Bioanal Chem 405 (2013), 8171-8184

[13] X. Ding, X. Zhan, X. Yuan, Z. Zhao and Y. Duan, Microfabricated Glow Discharge Plasma (MFGDP) for Ambient Desorption/Ionization Mass Spectrometry, Anal. Chem. 85 (2013), 9013-9020

[14] A. M. Bilgic, E. Voges, U. Engel and J. A. C. Broekaert, A low-power $2.45 \mathrm{GHz}$ microwave induced helium plasma source at atmospheric pressure based on microstrip technology, J. Anal. At. Spectrom. 15 (2000), 579-580

[15] U. Engel, A. M. Bilgiç, O. Haase, E. Voges and J. A. C. Broekaert, A MicrowaveInduced Plasma Based on Microstrip Technology and Its Use for the Atomic Emission Spectrometric Determination of Mercury with the Aid of the Cold-Vapor Technique, Anal. Chem. 72 (1999), 193-197

[16] S. Schermer, N. H. Bings, A. M. Bilgiç, R. Stonies, E. Voges and J. A. C. Broekaert, An improved microstrip plasma for optical emission spectrometry of gaseous species, Spectrochim. Acta Part B 58 (2003), 1585-1596

[17] I. Jiménez Zapata, P. Pohl, N. Bings and J. C. Broekaert, Evaluation and application of argon and helium microstrip plasma for the determination of mercury by the cold vapor technique and optical emission spectrometry, Anal Bioanal Chem 388 (2007), 1615-1623

[18] V. Červený, M. Horváth and J. A. C. Broekaert, Determination of mercury in water samples by electrochemical cold vapor generation coupled to microstrip microwave induced helium plasma optical emission spectrometry, Microchemical Journal 107 (2013), 10-16

[19] P. Pohl, I. Jimenéz Zapata, E. Voges, N. H. Bings and J. A. C. Broekaert, Comparison of the cold vapor generation using $\mathrm{NaBH} 4$ and $\mathrm{SnCl} 2$ as reducing agents and atomic emission spectrometry for the determination of $\mathrm{Hg}$ with a microstrip microwave induced argon plasma exiting from the wafer, Microchim Acta 161 (2008), 175-184

[20] P. Pohl, I. J. Zapata, N. H. Bings, E. Voges and J. A. C. Broekaert, Optical emission spectrometric determination of arsenic and antimony by continuous flow chemical hydride generation and a miniaturized microwave microstrip argon plasma operated inside a capillary channel in a sapphire wafer, Spectrochim. Acta Part B 62 (2007), 444453

[21] P. Pohl, I. J. Zapata and N. H. Bings, Optimization and comparison of chemical and electrochemical hydride generation for optical emission spectrometric determination of arsenic and antimony using a novel miniaturized microwave induced argon plasma exiting the microstrip wafer, Analytica Chimica Acta 606 (2008), 9-18

[22] A. Kadenkin and J. A. C. Broekaert, Studies with a miniaturized microwave induced plasma for element specific detection in gas chromatographic separations of halogenated hydrocarbons, J. Anal. At. Spectrom. 26 (2011), 1481-1487 
[23] P. Pohl, I. J. Zapata, M. A. Amberger, N. H. Bings and J. A. C. Broekaert, Characterization of a microwave microstrip helium plasma with gas-phase sample introduction for the optical emission spectrometric determination of bromine, chlorine, sulfur and carbon using a miniaturized optical fiber spectrometer, Spectrochim. Acta Part B 63 (2008), 415-421

[24] F. J. Andrade, J. T. Shelley, W. C. Wetzel, M. R. Webb, G. Gamez, S. J. Ray and G. M. Hieftje, Atmospheric Pressure Chemical Ionization Source. 1. Ionization of Compounds in the Gas Phase, Anal. Chem. 80 (2008), 2646-2653

[25] G. D. Schilling, J. T. Shelley, J. A. C. Broekaert, R. P. Sperline, M. B. Denton, C. J. Barinaga, D. W. Koppenaal and G. M. Hieftje, Use of an ambient ionization flowing atmospheric-pressure afterglow source for elemental analysis through hydride generation, J. Anal. At. Spectrom. 24 (2009), 34-40

[26] W. C. Wetzel, F. J. Andrade, J. A. C. Broekaert and G. M. Hieftje, Development of a direct current $\mathrm{He}$ atmospheric-pressure glow discharge as an ionization source for elemental mass spectrometry via hydride generation, J. Anal. At. Spectrom. 21 (2006), 750-756

[27] J. J. Ritter and N. K. Adams, Exponential dilution as a calibration technique, Anal. Chem. 48 (1976), 612-619

[28] T. Fujii, K. Iwase and P. C. Selvin, Mass spectrometric analysis of a $\mathrm{N}_{2} / \mathrm{H}_{2}$ microwave discharge plasma, International Journal of Mass Spectrometry 216 (2002), 169-175

[29] H. Uyama and O. Matsumoto, Synthesis of ammonia in high-frequency discharges, Plasma Chem Plasma Process 9 (1989), 13-24

[30] H. Uyama and O. Matsumoto, Synthesis of ammonia in high-frequency discharges. II. Synthesis of ammonia in a microwave discharge under various conditions, Plasma Chem Plasma Process 9 (1989), 421-432

[31] Y. Botchway George and M. Venugopalan, in Zeitschrift für Physikalische Chemie. 1980, vol. 120 , p. 103.

[32] J. N. Schaper, K. P. Pfeuffer, J. T. Shelley, N. H. Bings and G. M. Hieftje, Drop-onDemand Sample Introduction System Coupled with the Flowing Atmospheric-Pressure Afterglow for Direct Molecular Analysis of Complex Liquid Microvolume Samples, Anal. Chem. 84 (2012), 9246-9252

[33] W. C. Wetzel, J. A. C. Broekaert and G. M. Hieftje, Determination of arsenic by hydride generation coupled to time-of-flight mass spectrometry with a gas sampling glow discharge, Spectrochim. Acta Part B 57 (2002), 1009-1023

[34] K. P. Pfeuffer, J. T. Shelley, S. J. Ray and G. M. Hieftje, Visualization of mass transport and heat transfer in the FAPA ambient ionization source, J. Anal. At. Spectrom. 28 (2013), 379-387 
Figures:

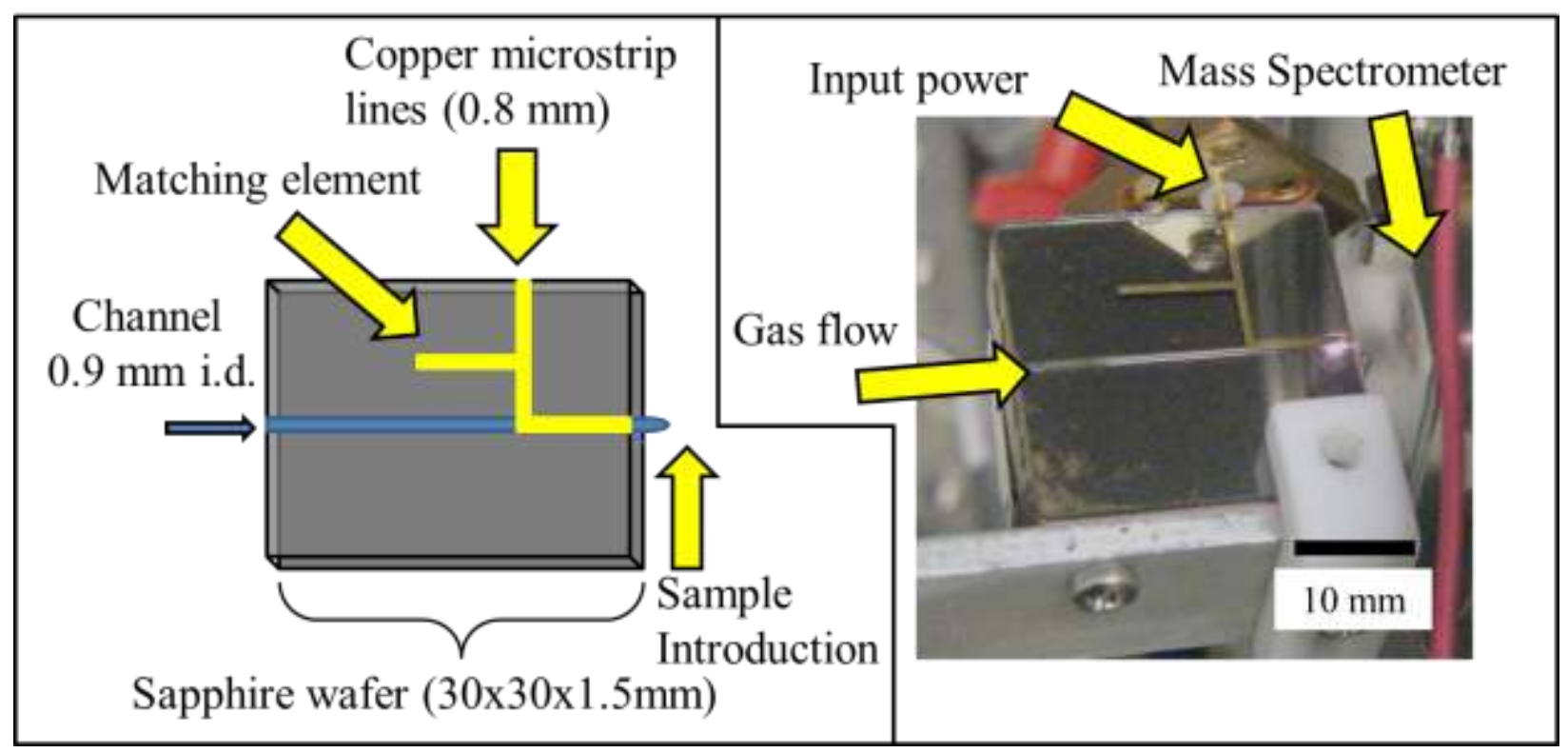

Figure 1 Schematic diagram of microwave microstrip plasma (MSP) used in this work (left) and a white-light image of the source (right),which shows it sitting in front of the mass spectrometer. Scale bar represents $10 \mathrm{~mm}$ in image on right. 


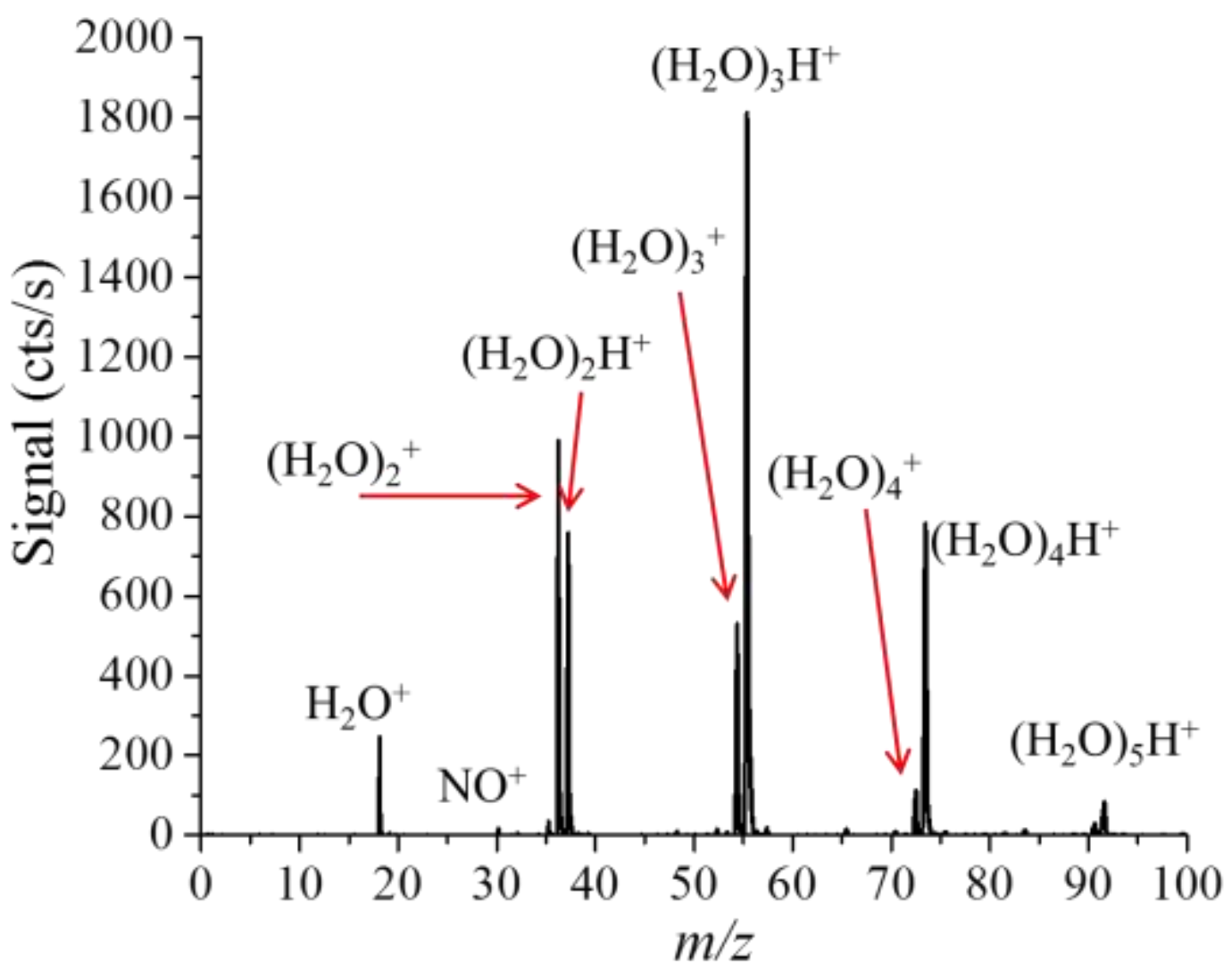

Figure 2. Background mass spectrum from microstrip plasma, which demonstrates that the major ions are water clusters both ionized directly and protonated. 


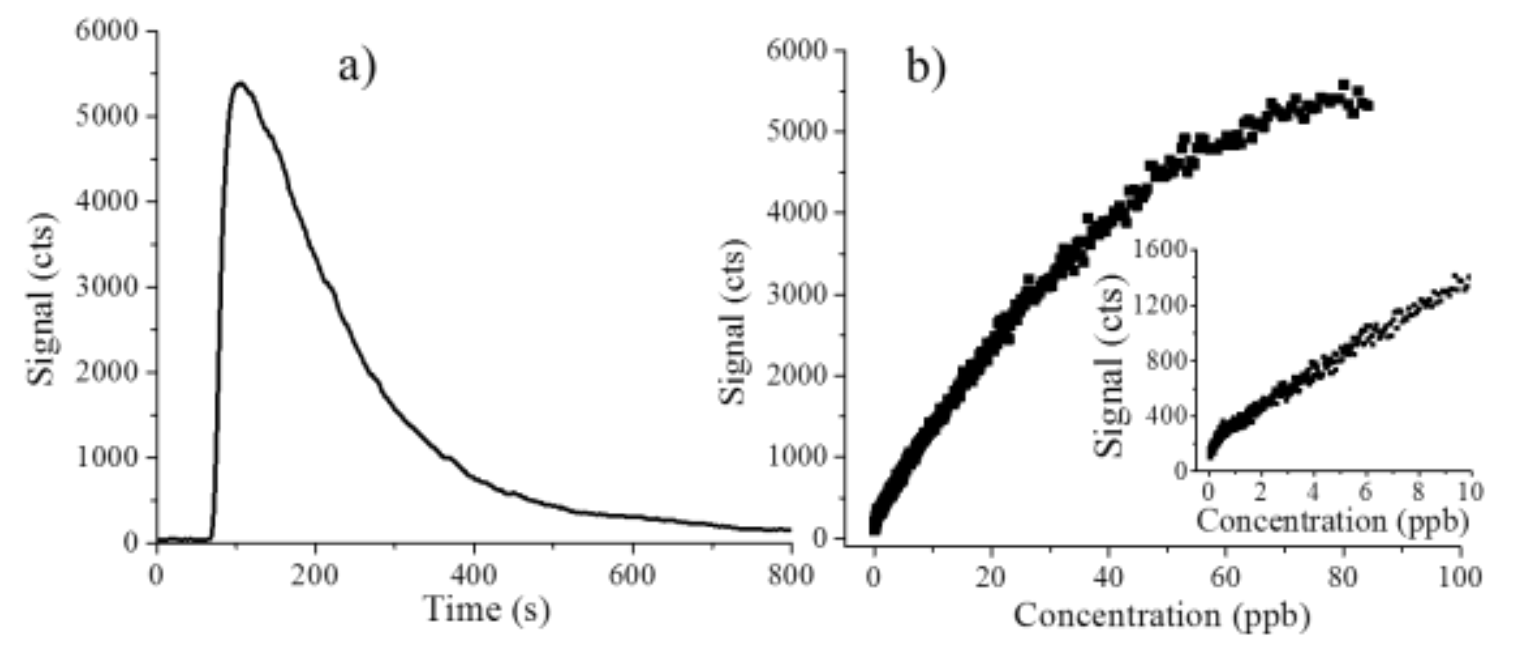

Figure 3. Organic vapor introduction via an exponential-dilution chamber. a) Time trace for protonated acetonitrile; injection occurred at 60 seconds. b) Calibration curve for acetonitrile generated from $\mathrm{a}$; inset shows concentration range ( $\mathrm{ppb} \mathrm{v} / \mathrm{v})$ that was utilized for limits of detection. 
Table 1. Compounds tested, ion detected, amount injected and limits of detection for volatile organic compounds introduced into the MSP from an exponential dilution flask

\begin{tabular}{|c|c|c|c|}
\hline Compound & $\begin{array}{c}\text { Ion } \\
\text { Detected }\end{array}$ & $\begin{array}{c}\text { Amount } \\
\text { Injected }(\boldsymbol{\mu g})\end{array}$ & LOD vol/vol (ppm) \\
\hline Ethanol & $(\mathrm{M}+\mathrm{H})^{+}$ & 180 & 0.36 \\
\hline Ethyl Acetate & $(\mathrm{M}+\mathrm{H})^{+}$ & 350 & 1.6 \\
\hline Chloroform & $\mathrm{M}^{+}$ & 990 & 7.4 \\
\hline Benzene & $\mathrm{M}^{+}$ & 310 & 0.12 \\
\hline Acetonitrile & $(\mathrm{M}+\mathrm{H})^{+}$ & 160 & 0.042 \\
\hline Acetone & $(\mathrm{M}+\mathrm{H})^{+}$ & 580 & \\
\hline
\end{tabular}




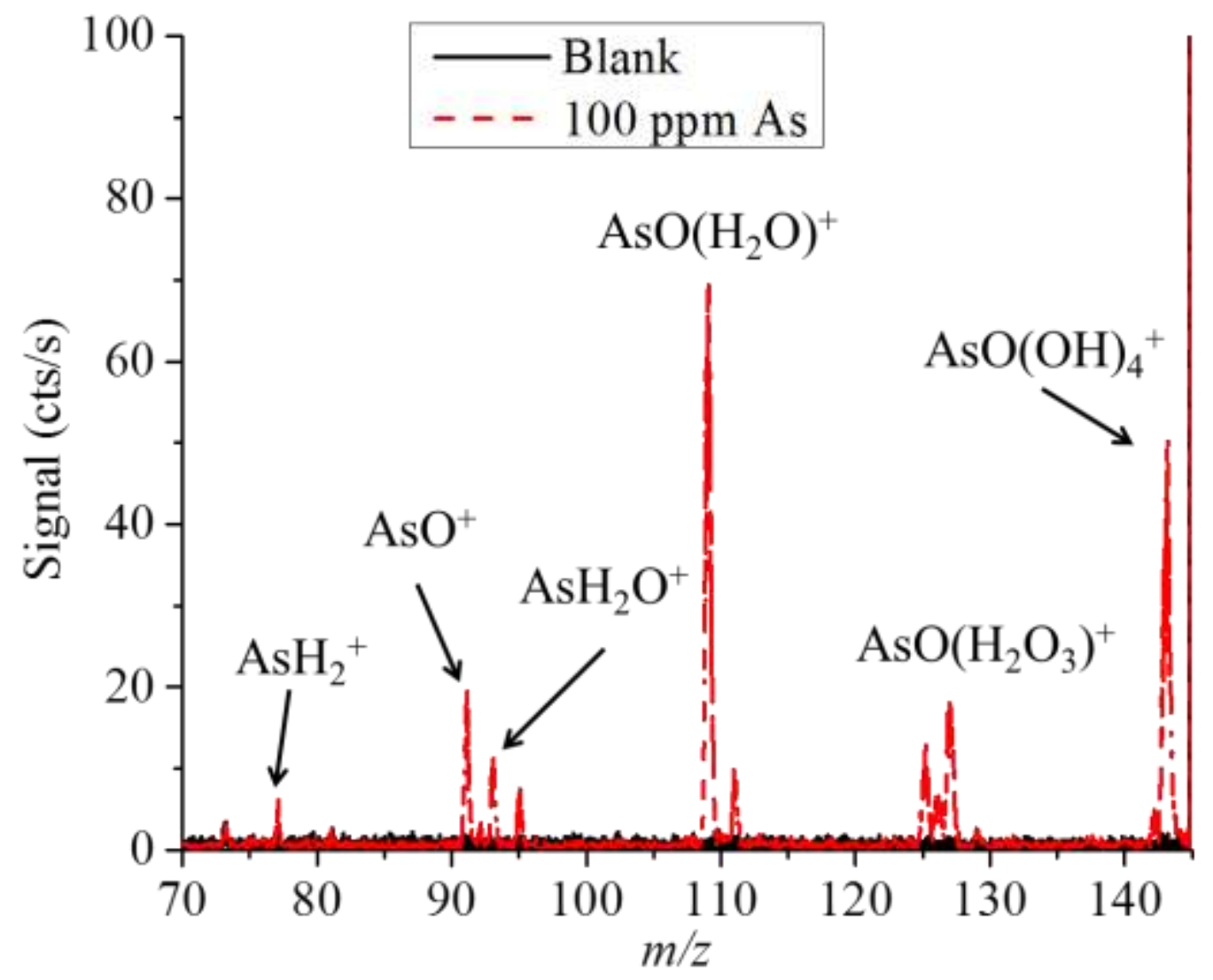

Figure 4. Spectra from introduction of arsenic-containing solution (100 ppm) into the hydride-generation - microstrip plasma system. 


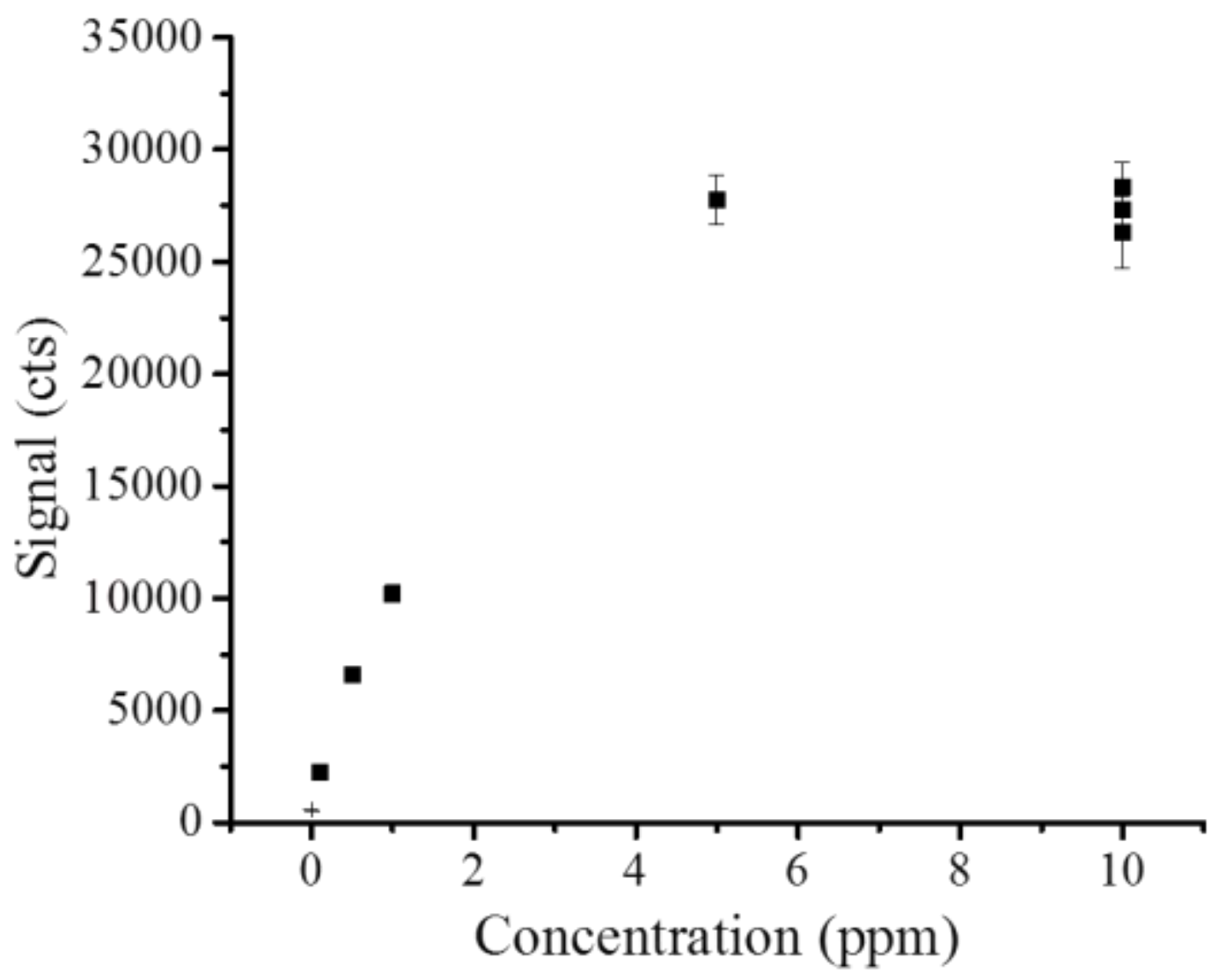

Figure 5. Calibration curve for arsenic $\left(\mathrm{AsO}\left(\mathrm{H}_{2} \mathrm{O}\right)^{+}\right.$peak) with the hydride-generation system coupled to the MSP afterglow. Significant roll-off occurs above $5 \mathrm{ppm}$. The $10 \mathrm{ppm}$ concentration was run 3 times to determine reproducibility. 
Table 2. Elements, species detected, and limits of detection for hydride generation coupled to the MSP afterglow_mass spectrometry setup.

\begin{tabular}{|c|c|c|}
\hline Element & Species Detected & $\begin{array}{c}\text { Solution LOD } \\
(\mathrm{ppb})\end{array}$ \\
\hline Tin & $\mathrm{Sn}\left(\mathrm{H}_{3} \mathrm{O}\right)^{+}$ & 56 \\
\hline Arsenic & $\mathrm{AsO}(\mathrm{OH})_{4}^{+}$ & 36 \\
\hline Antimony & $\mathrm{SbH}_{3}^{+}$ & 120 \\
\hline
\end{tabular}




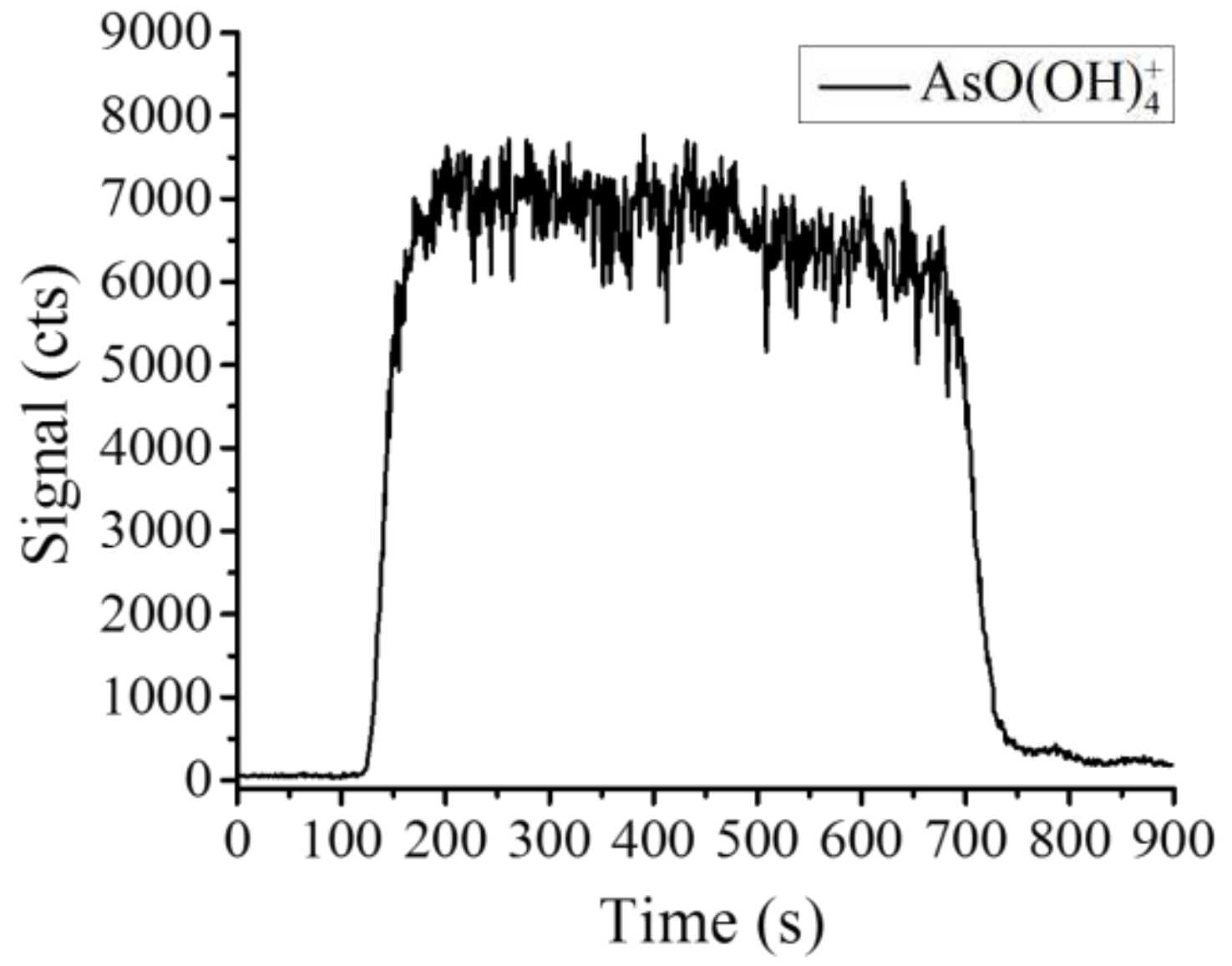

Figure 6. Washout curve for arsenic with the hydride-generation setup. Based on a 90/10\% criterion, the rise time was $30 \mathrm{~s}$ and the fall-time was $50 \mathrm{~s}$. 

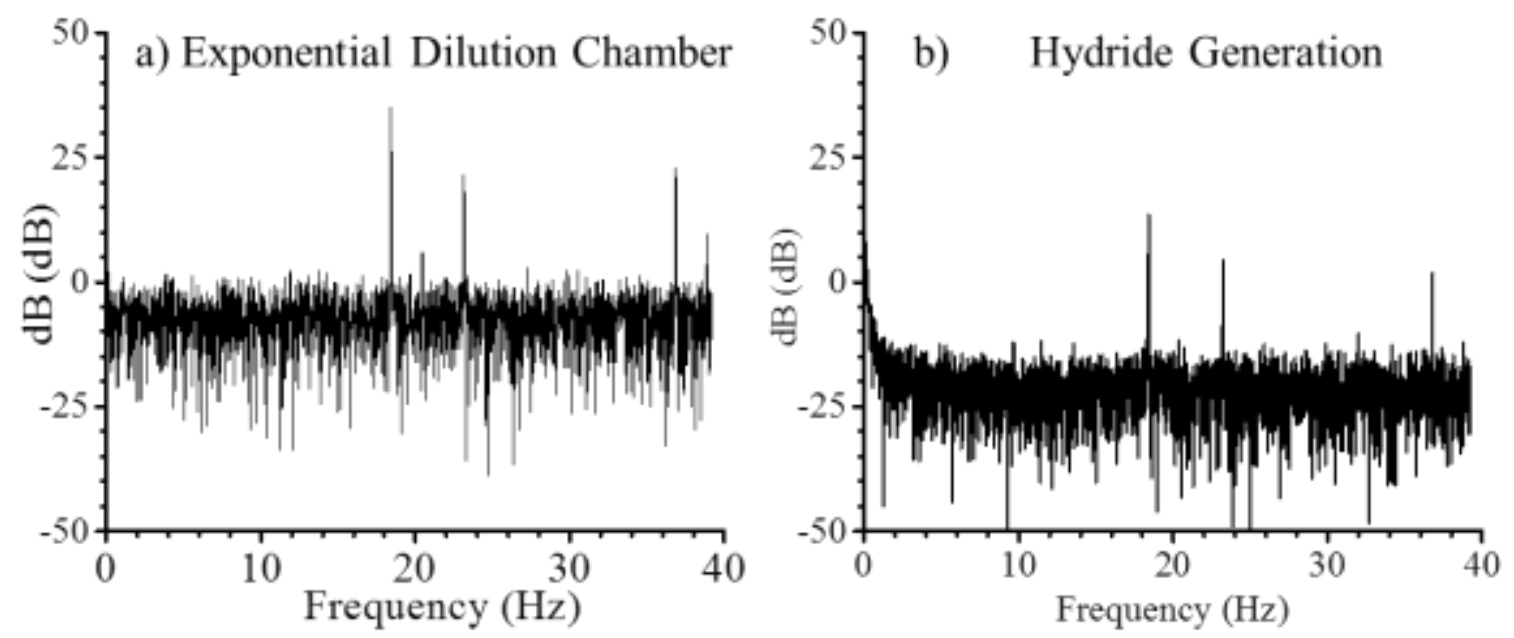

Figure 7. Noise power spectra for the two sample-introduction systems coupled to the MSP afterglow: a) exponential dilution chamber, with the protonated water $\operatorname{dimer}\left(\mathrm{H}_{2} \mathrm{O}\right)_{2} \mathrm{H}^{+}$being monitored, and b) hydride generation system monitoring the $\mathrm{As}(\mathrm{OH})_{4}$ channel. 


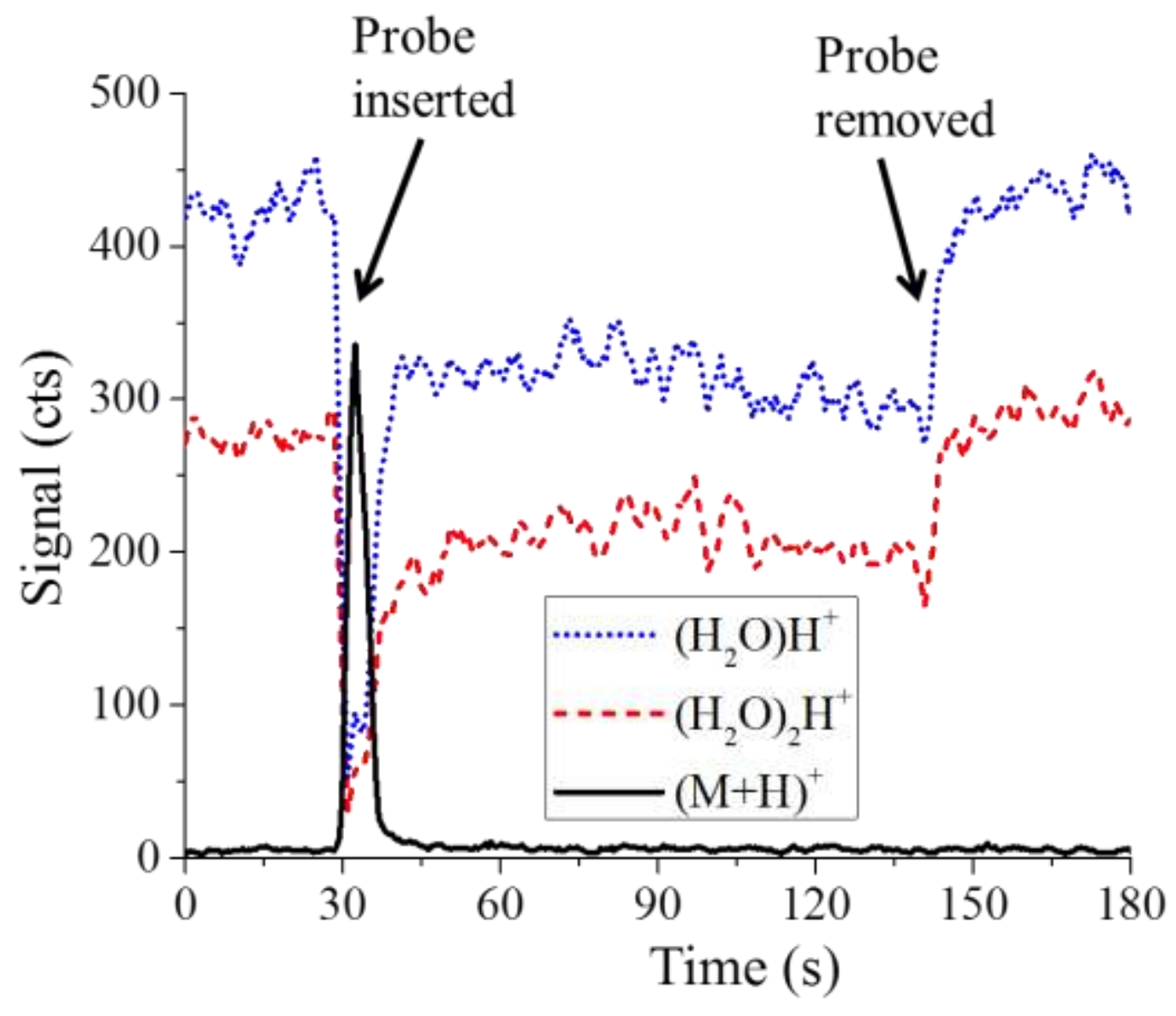

Figure 8. Time trace for protonated acetaminopen and two major reagent ions during probe introduction of a solid sample residue into the MSP afterglow. 